\title{
Additive suppression of tonic-clonic seizures in mice receiving the combination of carbamazepine, phenobarbital and valproate
}

\author{
Katarzyna Załuska', Paweł Marzęda', Hubert Bojar², Aleksandra Walczak', \\ Jarosław Chmielewski ${ }^{3}$, Paula Wróblewska-Łuczka', Jarogniew J Łuszczki ${ }^{1,4}$ \\ ${ }^{1}$ Department of Pathophysiology, Medical University of Lublin, Poland \\ ${ }^{2}$ Department of Toxicology and Food Safety, Institute of Rural Health, Lublin, Poland \\ ${ }^{3}$ Institute of Environmental Protection - National Research Institute in Warsaw, Poland \\ ${ }^{4}$ Isobolographic Analysis Laboratory, Institute of Rural Health, Lublin, Poland
}

Załuska K, Marzęda P, Bojar H, Walczak A, Chmielewski J, Wróblewska-Łuczka P, Łuszczki JJ. Pre-Clin Clin Res. 2019; 13(2): 72-75. doi: 10.26444/ jpccr/108899

\section{Abstract}

Introduction. Polytherapy with three antiepileptic drugs (AEDs) is used in patients who have seizure episodes classified by physicians and neurologists as refractory or drug-resistant. Although doctors can prescribe these patients 25 various AEDs, no algorithms are available which allow them to choose the most efficacious combinations of AED.

Objective. The aim of this study was to isobolographically classify interaction among three classical AEDs (carbamazepine, phenobarbital and valproate), applied in the fixed-ratio combination of 1:1:1, in the maximal electroshock-induced seizures in mice.

Materials and method. The anti-seizure activity of the mixture of carbamazepine, phenobarbital and valproate (in the fixed-ratio of 1:1:1) was determined in maximal electroshock-induced seizures - an experimental model of tonic-clonic seizures in mice using type I isobolographic analysis.

Results. The mixture of carbamazepine, phenobarbital and valproate (in the fixed-ratio of 1:1:1) produced additive interaction in the maximal electroshock-induced seizure model in mice. The experimentally-derived median effective dose (ED50 exp value) for the mixture was $94.35 \mathrm{mg} / \mathrm{kg}$, whereas the theoretically additive median effective dose (ED50 add value) amounted to $116.77 \mathrm{mg} / \mathrm{kg}$.

Conclusions. Although the combination of carbamazepine, phenobarbital and valproate exerted additivity in the mouse maximal electroshock-induced seizure model, it could be recommended for the treatment of patients, if the results of this study would be directly transposed to clinical settings.

\section{Key words}

drug Interactions, carbamazepine, isobolographic analysis, phenobarbital, valproate

\section{INTRODUCTION}

Despite the wide range of antiepileptic drugs (AEDs) currently available for the treatment of epilepsy, there is still no consensus on which drug should be preferentially used as the first-line AED in the treatment of epilepsy [1-3]. Some AEDs occur "virtually" inactive in the treatment of seizures and the patients need to combine two or three AEDs to substantially limit seizure activity and /or frequency [4, 5]. The process of choice of AEDs and joining them together in one combination is performed by clinicians based on their clinical experience. However, this process is biased by professional experience of doctors prescribing specific AEDs $[4,5]$. This is the reason that clinical experience plays a crucial role in the selection of AEDs. To help clinicians make the right decision about the selection of efficacious AEDs, isobolographic analysis of interaction in preclinical studies on animals is recommended [6-9].

Previously, it has been documented that some three-drug mixture of AEDs produced synergistic, additive or even antagonistic interactions in the mouse maximal electroshockinduced seizure model (Tab. 1).

Address for correspondence: Jarogniew J. Łuszczki, Isobolographic Analysis Laboratory, Institute of Rural Health, Lublin, Poland

E-mail: jarogniew.luszczki@umlub.pl

Received: 10 April 2019; accepted: 15 May 2019; first published: 30 May 2019
Table 1. Characteristics of interactions for the studied three-drug combinations

\begin{tabular}{lcc}
\hline Combination & Type of interaction & References \\
\hline oxcarbazepine + pregabalin + topiramate & supra-additive & {$[7]$} \\
\hline lacosamide + carbamazepine + valproate & infra-additive & {$[10]$} \\
\hline lacosamide + carbamazepine + lamotrigine & additive & {$[11]$} \\
\hline lacosamide + carbamazepine + phenobarbital & additive & {$[12]$} \\
\hline lacosamide + lamotrigine + phenobarbital & additive & {$[13]$} \\
\hline carbamazepine + phenobarbital + topiramate & supra-additive & {$[14]$} \\
\hline phenobarbital + phenytoin + pregabalin & supra-additive & {$[15]$} \\
\hline
\end{tabular}

However, the types of interactions determined in animals in the mouse tonic-clonic seizure test cannot be predicted theoretically. In the 90 s of the $20^{\text {th }}$ century, a suggestion of combining AEDs with various molecular mechanisms of action became a recommendation for physicians and neurologists to combine AEDs when treating the patients with refractory epilepsy $[8,16]$.

Considering the above-mentioned facts and results published earlier from the mouse tonic-clonic seizure model, it was evident that theoretical presumptions about the combination of AEDs with different molecular mechanisms of action are sometimes contradictory to the results obtained experimentally. Of note, when considering the combination 
of lacosamide with carbamazepine and valproate, the mixture should theoretically exert supra-additive (synergistic) interaction due to various molecular mechanisms of action of the AEDs. Unfortunately, the mixture of lacosamide with carbamazepine and valproate produced infra-additivity (antagonism) in the mouse maximal electroshock-induced seizure model [10].

Because theoretical presumptions sometimes differed from those experimentally confirmed in the mouse tonicclonic seizure model, in this study an attempt was made to conduct experiments in this seizure model to determine the type of interaction occurring among carbamazepine (CBZ), phenobarbital (PB) and valproate (VPA) using type I isobolographic analysis of interaction, as recommended earlier [7, 10, 17-19]. Theoretically, the combination of CBZ, PB and VPA should produce synergy in mice due to various molecular mechanisms of action of the studied AEDs. Undoubtedly, the synergistic combination of CBZ, PB and VPA, confirmed in preclinical studies, can offer a new treatment option for some epileptic patients, especially if the AEDs belong to the first generation of AEDs [20].

\section{MATERIALS AND METHOD}

Animals. Experiments on adult male albino Swiss mice weighing 22-26 g were performed in accordance with the ARRIVE guidelines and Polish law concerning experimentation on laboratory animals. All experimental protocols performed in this study were approved by the Second Local Ethics Committee at the University of Life Sciences in Lublin, Poland. After acclimatization to laboratory conditions, the animals were randomly assigned to experimental groups consisting of eight mice each.

Drugs. All three AEDs (CBZ, PB and VPA) were purchased from Sigma-Aldrich (Poznań, Poland). Both CBZ and PB were dispersed in 1\% solution of Tween 80 (Sigma-Aldrich, Poznań, Poland), whereas VPA was directly dissolved in distilled water. The experimental animals received intraperitoneal injections with $\mathrm{CBZ}$ at $30 \mathrm{~min}, \mathrm{~PB}$ - at $60 \mathrm{~min}$, and VPA - at $30 \mathrm{~min}$, before electrical induction of seizures in the maximal electroshock-induced seizure test, as presented earlier $[10-12,14,15]$.

Maximal electroshock-induced seizures. In the maximal electroshock-induced seizure test, an alternating current (25 mA, $500 \mathrm{~V}, 50 \mathrm{~Hz}, 0.2 \mathrm{~s}$ stimulus duration) delivered via ear-clip electrodes was used to evoke seizure activity in the animals. The respective groups of mice were injected with different doses of CBZ, PB or VPA (either separately or in combination) and, at the respective pretreatment times, were subjected to maximal electroconvulsions. The mice protected from tonic-clonic seizures out of eight animals in each experimental group allowed to construct doseresponse effect line, according to the log-probit method [21]. The anticonvulsant effects of CBZ, PB and VPA (when administered separately and in the three-drug mixture at the fixed-ratio of 1:1:1) were expressed as the median effective doses $\left(\mathrm{ED}_{50}\right.$ in $\left.\mathrm{mg} / \mathrm{kg}\right)$ for the AEDs administered separately and the experimental median effective dose $\left(\mathrm{ED}_{50 \exp }\right.$ in $\mathrm{mg} /$ $\mathrm{kg}$ ) for the three-drug mixture, as presented elsewhere [7, $15,22-25]$.
Isobolography and statistics. Interaction produced by the mixture of CBZ, PB and VPA at the fixed-ratio of 1:1:1 was analyzed isobolographically, as recommended earlier [2628]. Calculation of median additive dose $\left(\mathrm{ED}_{50 \text { add }}\right)$ along with experimental determination of median experimental dose $\left(\mathrm{ED}_{50 \text { exp }}\right)$ for the three-drug mixture (at the fixed-ratio combination of $1: 1: 1)$ was required to statistically verified both values, as presented elsewhere $[10,13]$. Of note, the unpaired Student's $t$-test was used to statistically compare the $\mathrm{ED}_{50 \text { exp }}$ and $\mathrm{ED}_{50 \text { add }}$ values for the three-drug mixture, as described elsewhere $[12,17]$. Level of statistical significance was established at $P<0.05$. Statistical analysis of data was performed with commercially available GraphPad Prism (version 7.0 for Windows; GraphPad Software, San Diego, CA, USA).

\section{RESULTS}

Anticonvulsant effects of the three-drug mixture and isobolographic analysis of interaction. The three AEDs (CBZ, PB and VPA) when combined together in the mixture at the fixed-ratio combination of 1:1:1 protected the animals from the maximal electroshock-induced tonic-clonic seizures, and the experimentally-denoted median effective dose $\left(\mathrm{ED}_{50 \text { exp }}\right.$ value) amounted to $94.35 \pm 9.85 \mathrm{mg} / \mathrm{kg}$ (Figure $1 \mathrm{~A}-\mathrm{C})$. The theoretically additive median effective dose $\left(\mathrm{ED}_{50 \text { add }}\right.$ value) was $116.77 \pm 6.78 \mathrm{mg} / \mathrm{kg}$ (Fig. $\left.1 \mathrm{~A}-\mathrm{C}\right)$. With isobolographic analysis of interaction, the $\mathrm{ED}_{50 \text { exp }}$ value for the three-drug mixture of CBZ, PB and VPA did not differ significantly from the $\mathrm{ED}_{50 \text { add }}$ value (Fig. $1 \mathrm{~A}-\mathrm{C}$ ); thus, the observed interaction was additive in the mouse model of tonic-clonic seizures.

\section{DISCUSSION}

In the study it was found that the three classical AEDs (CBZ, $\mathrm{PB}$ and VPA) when combined together exerted an additive interaction with a slight tendency towards supra-additivity in the mouse tonic-clonic seizure model. Type I isobolographic analysis confirmed that the dose-response effects of all three AEDs were parallel to each other. Despite a tendency towards supra-additivity observed with isobolography, statistical analysis with Student's t-test revealed that the analyzed values did not reach significance; therefore the interaction was classified as additive, according to Tallarida [29].

The observed additivity in the mouse maximal electroshock-induced seizure model for the combination of CBZ with $\mathrm{PB}$ and VPA, was in agreement with interactions observed previously in animals. For instance, it has been reported that the combination of CBZ with $\mathrm{PB}$ and LCM in the mouse model of tonic-clonic seizures exerted additive interaction [12]. In contrast, OXC with PGB and TPM exerted supra-additive interaction in the mouse maximal electroshock-induced seizure model [7]. Similarly, PB with PHT and PGB exerted synergy in the tonic-clonic seizure model in mice [15]. The combination of CBZ with $\mathrm{PB}$ and TPM also exerted synergy in the mouse maximal electroshock-induced seizure model [14].

Considering the latter three-drug combination and comparing the results from the current study, one can observe that the replacement of TPM with VPA changes 


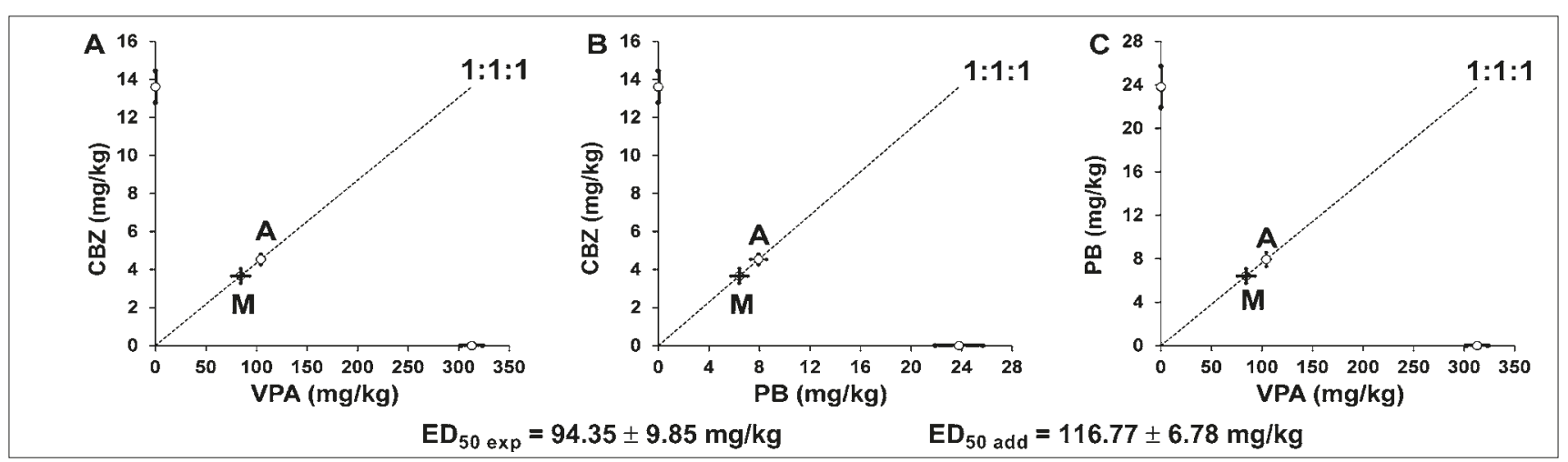

Figure 1A-1C. Isobolograms displaying additive interaction among carbamazepine (CBZ), phenobarbital (PB) and valproate (VPA) in the maximal electroshock-induced seizure test in mice.

Median effective doses ( $E_{50} \pm$ S.E.M.) of CBZ, PB and VPA (when administered alone) are plotted on abscissa and ordinate, respectively. Point A reflects the ED ${ }_{50 \text { add }}$ value, point $\mathrm{M}$ indicates the $\mathrm{ED}_{50}$ value. Statistical analysis with unpaired Student's $t$-test revealed that both, $\mathrm{ED}_{50 \exp }$ and $\mathrm{ED}_{50 \text { add }}$ values did not differ significantly. Interaction among CBZ, PB and VPA was additive in the maximal electroshock-induced seizure test in mice.

the type of interaction for the combination of CBZ with $\mathrm{PB}$ from supra-additive to additive. Similarly, the combination of CBZ with PB and LCM was additive in the mouse maximal electroshock-induced seizure model [12]. In such cases, both VPA and LCM contribute to the additive interaction of the combinations of CBZ with $\mathrm{PB}$, and VPA or LCM in the mouse maximal electroshock-induced seizure model. On the contrary, the replacement of VPA with TPM produced synergistic interaction in animals subjected to the maximal electroshock-induced seizure test. It can be suggested that VPA and LCM, through the action on sodium channels, exerted additivity, whereas TPM through multiple molecular mechanisms of action potentiated significantly the interaction of CBZ with $\mathrm{PB}$ in the mouse tonic-clonic seizure model. Although this hypothesis is speculative, it can explain the observed types of interactions among three AEDs.

It is worth mentioning that in the mouse maximal electroshock-induced seizure test, one can readily determine and assess the type of interactions occurred among AEDs in terms of suppression of tonic-clonic seizures and partial convulsions. As regards the combinations of $\mathrm{CBZ}, \mathrm{PB}$ and VPA, it should be clearly stated that all these classical AEDs are used clinically to treat epilepsy patients with tonic-clonic seizures and partial-onset convulsions. Moreover, it should be emphasized that all three AEDs are first-line AEDs used in patients with generalized tonic-clonic seizures [20].

Another aspect of interaction occurring among AEDs should be discussed here. Interactions occurring among three AEDs should be evaluated with respect to their twodrug combinations. Previously, it has been reported that the combination of CBZ with PB exerted "purely additive" interaction in the mouse maximal electroshock-induced seizure model [30]. A similar situation has been observed for the combination of VPA with CBZ or PB, because the interaction among these AEDs was also "purely additive" in the mouse maximal electroshock-induced seizure model [31]. Considering the type of interactions exerted by these two-drug combinations, one could suggest that the observed interaction would also be additive in the mouse maximal electroshock-induced seizure model. However, when considering molecular mechanisms of action of the studied three AEDs, one could expect supra-additive interaction because all the studied AEDs possessed various molecular mechanisms of action, and they should theoretically produce synergistic interaction in the experimental animals [8]. Furthermore, calculation of interaction index as a ratio of $\mathrm{ED}_{50 \text { exp }}$ and $\mathrm{ED}_{50 \text { add }}$ values, revealed that the interaction of CBZ, PB and VPA can be classified as additive because the interaction index value amounted to 0.81 . Generally, the additive interactions are documented if their interaction indices ranged from $0.7-1.3$ [28]. Of note, the calculation of interaction index is an alternative way to classify the interaction occurring between AEDs. Although this method is supplementary to statistical analysis of data with Student's t-test, there is no doubt that statistical comparison of $\mathrm{ED}_{50 \exp }$ and $\mathrm{ED}_{50 \text { add }}$ values is superior to the calculation of interaction index in experiments evaluating the types of interactions among AEDs. This was the reason that the Student's $t$-test was performed in this study as a unique indicator to properly classify interaction.

It should be stressed that this study determined a potential for AEDs administered in combination to produce acute adverse effects with respect to impairment of motor coordination, deficits in long-term memory, and attenuation of skeletal muscular strength (results not shown). Experiments conducted on animals in the chimney, stepthough passive avoidance and grip-strength tests revealed that the combination of $\mathrm{CBZ}$ with $\mathrm{PB}$ and VPA, administered in doses of AEDs reflecting the $\mathrm{ED}_{50 \exp }$ value, exerted no acute adverse effects. The combination did not exert any significant changes in the normal behaviour of experimental animals. It can be confirmed that the combination of CBZ, PB and VPA did not produce acute impairment in motor coordination, deficits in long-term memory, or changes in skeletal muscular strength in mice. Thus, the combination seems to be safe enough to be recommended for use in clinical practice for epileptic patients.

It should be explained that in this study only acute adverse effects were tested after a single administration of the mixture of three AEDs to animals. It should also be stressed that after the single administration of AEDs, the three-drug mixture was unable to significantly change metabolic transformation of co-administered AEDs in the liver. The authors are aware of the fact that during chronic administration of AEDs, some pharmacokinetic changes related to AEDs' contents may occur as a result of inducing or inhibiting cytochrome P450 isoenzymes. In chronic experiments, pharmacokinetic interactions among AEDs occur more frequently than those in acute studies. 
The main limitation in this study was lack of pharmacokinetic verification of total brain concentrations of AEDs administered in triple combination. Such verification could unequivocally confirm whether or not the observed interaction was pharmacodynamic in mice. Of note, any pharmacokinetic contribution to the observed interaction is unlikely because doses of particular classical AEDs in the mixture were low enough to produce significant changes in total brain AED concentrations. Although total brain content of AEDs was not measured in mice, it cannot be entirely excluded that the observed additivity in the mouse maximal electroshock-induced seizure model had, at least in part, a pharmacokinetic contribution to the interaction occurring among the three AEDs when combined together. The AED concentrations were not evaluated in this study for three reasons:

1) the interaction observed in the mouse maximal electroshock-induced seizure model was additive;

2) the doses of particular drugs used in the mixture were "substantially" low;

3 ) the ethical rule of " $3 \mathrm{Rs}$ " based on the reduction of animals exposed to experimental conditions.

All three reasons prompted the authors to accept the hypothesis that CBZ, PB and VPA were unable to interact pharmacokinetically in mice when used in triple combination in doses reflecting the $\mathrm{ED}_{50 \exp }$ from the maximal electroshockinduced seizure test.

\section{CONCLUSIONS}

1. The three-drug combination of CBZ with PB and VPA exerted additive interaction in the mouse model of maximal electroshock-induced seizures.

2. The combination of CBZ with PB and VPA may occur to be advantageous for epilepsy patients who have inadequately controlled seizures.

\section{REEFERENCES}

1.Brodie MJ. Pharmacological treatment of drug-resistant epilepsy in adults: a practical guide. Curr Neurol Neurosci Rep. 2016;16:82.

2.Brodie MJ, Sills GJ. Combining antiepileptic drugs--rational polytherapy? Seizure. 2011;20:369-375.

3. Brodie MJ, Barry SJ, Bamagous GA, Norrie JD, Kwan P. Patterns of treatment response in newly diagnosed epilepsy. Neurology. 2012;78:1548-1554.

4. Stephen LJ, Brodie MJ. Seizure freedom with more than one antiepileptic drug. Seizure. 2002;11:349-351.

5.Stephen LJ, Forsyth M, Kelly K, Brodie MJ. Antiepileptic drug combinations--have newer agents altered clinical outcomes? Epilepsy Res. 2012;98:194-198.

6. Matsumura N, Nakaki T. Isobolographic analysis of the mechanisms of action of anticonvulsants from a combination effect. Eur J Pharmacol. 2014;741:237-246.

7.Załuska K, Kondrat-Wróbel MW, Panasiuk-Poterek AN, Marzęda P, Walczak A, Wróblewska-Łuczka P, et al. Synergy among oxcarbazepine, pregabalin and topiramate in the mouse maximal electroshockinduced seizure test - an isobolographic analysis. J Pre-Clin Clin Res. 2018;12:111-116.

8. Deckers CL, Czuczwar SJ, Hekster YA, Keyser A, Kubova H, Meinardi H, et al. Selection of antiepileptic drug polytherapy based on mechanisms of action: the evidence reviewed. Epilepsia. 2000;41:1364-1374.

9. Blaszczyk B, Miziak B, Czuczwar P, Wierzchowska-Cioch E, Pluta $\mathrm{R}$, Czuczwar SJ. A viewpoint on rational and irrational fixed-drug combinations. Exp Rev Clin Pharmacol. 2018:1-11.
10. Kondrat-Wróbel MW, Załuska K, Walczak A, Panasiuk-Poterek AN, Gut-Lepiech A, Wróblewska-Łuczka P, et al. Antagonistic interaction of lacosamide with carbamazepine and valproate in the mouse tonicclonic seizure model. Health Probl Civil. 2019;13:92-98.

11. Kondrat-Wrobel MW, Luszczki JJ. Additive interaction for three-drug combination of carbamazepine, lacosamide and lamotrigine against maximal electroshock-induced seizures - a type I isobolographic analysis. Eur J Clin Exp Med. 2017;15:303-309.

12. Kondrat-Wrobel MW, Luszczki JJ. Interaction of three-drug combination of lacosamide, carbamazepine and phenobarbital in the mouse maximal electroshock-induced seizure model - an isobolographic analysis. Health Probl Civil. 2016;10:55-61.

13. Kondrat-Wrobel MW, Luszczki JJ. Isobolographic additivity among lacosamide, lamotrigine and phenobarbital in a mouse tonic-clonic seizure model. Adv Clin Exp Med. 2018;27:881-886.

14.Luszczki JJ. Isobolographic analysis of interaction for three-drug combination of carbamazepine, phenobarbital and topiramate in the mouse maximal electroshock-induced seizure model. Pharmacology. 2016;97:259-264.

15. Luszczki JJ, Mazurkiewicz LP, Wroblewska-Luczka P, Wlaz A, Ossowska G, Szpringer M, et al. Combination of phenobarbital with phenytoin and pregabalin produces synergy in the mouse tonic-clonic seizure model: An isobolographic analysis. Epilepsy Res. 2018;145:116-122.

16. Perucca E. Pharmacological principles as a basis for polytherapy. Acta Neurol Scand Suppl. 1995;162:31-34.

17. Luszczki JJ. Isobolographic analysis of interaction between drugs with nonparallel dose-response relationship curves: a practical application. Naunyn Schmiedebergs Arch Pharmacol. 2007;375:105-114.

18. Luszczki JJ, Zagaja M, Miziak B, Kondrat-Wrobel MW, Zaluska K, Wroblewska-Luczka P, et al. Beneficial combination of lacosamide with retigabine in experimental animals: an isobolographic analysis. Pharmacology. 2018;101:22-28.

19.Zolkowska D, Zagaja M, Miziak B, Kondrat-Wrobel MW, Zaluska K, Florek-Luszczki $\mathrm{M}$, et al. Isobolographic assessment of interactions between retigabine and phenytoin in the mouse maximal electroshockinduced seizure model and chimney test. Health Probl Civil. 2016;10:5459.

20. National Clinical Guideline Centre. National Institute for Health and Clinical Excellence: Guidance. The Epilepsies: the diagnosis and management of the epilepsies in adults and children in primary and secondary care: pharmacological update of clinical guideline 20. London: Royal College of Physicians (UK); National Clinical Guideline Centre; 2012.

21. Litchfield JT, Jr., Wilcoxon F. A simplified method of evaluating doseeffect experiments. J Pharmacol Exp Ther. 1949;96:99-113.

22. Luszczki JJ. Interactions between pregabalin and phenobarbital in the mouse maximal electroshock-induced seizure model: an isobolographic analysis. J Pre-Clin Clin Res. 2009;3:103-109.

23. Luszczki JJ, Ratnaraj N, Patsalos PN, Czuczwar SJ. Pharmacodynamic and pharmacokinetic interaction studies of loreclezole with felbamate, lamotrigine, topiramate, and oxcarbazepine in the mouse maximal electroshock seizure model. Epilepsia. 2005;46:344-355.

24.Luszczki JJ, Ratnaraj N, Patsalos PN, Czuczwar SJ. Characterization of the anticonvulsant, behavioral and pharmacokinetic interaction profiles of stiripentol in combination with clonazepam, ethosuximide, phenobarbital, and valproate using isobolographic analysis. Epilepsia. 2006;47:1841-1854.

25.Luszczki JJ, Ratnaraj N, Patsalos PN, Czuczwar SJ. Isobolographic analysis of interactions between loreclezole and conventional antiepileptic drugs in the mouse maximal electroshock-induced seizure model. Naunyn Schmiedebergs Arch Pharmacol. 2006;373:169-181.

26. Loewe $S$. The problem of synergism and antagonism of combined drugs. Arzneimittelforschung. 1953;3:285-290.

27. Tallarida RJ. Revisiting the isobole and related quantitative methods for assessing drug synergism. J Pharmacol Exp Ther. 2012;342:2-8.

28. Gessner PK. Isobolographic analysis of interactions: an update on applications and utility. Toxicology. 1995;105:161-179.

29. Tallarida RJ. Drug synergism and dose-effect data analysis. New York: Chapman and Hall/CRC 2000.

30. Bourgeois BF, Wad N. Combined administration of carbamazepine and phenobarbital: effect on anticonvulsant activity and neurotoxicity. Epilepsia. 1988;29:482-487.

31. Bourgeois BF. Anticonvulsant potency and neurotoxicity of valproate alone and in combination with carbamazepine or phenobarbital. Clin Neuropharmacol. 1988;11:348-359. 